\title{
Conjunctival inflammation in the chronic phase of Stevens-Johnson syndrome
}

\author{
Satoshi Kawasaki, Kohji Nishida, Chie Sotozono, Andrew J Quantock, Shigeru Kinoshita
}

\begin{abstract}
Aims-To understand the immunopathogenesis of the corneal conjunctivalisation in Stevens-Johnson syndrome.

Methods-Conjunctivalised corneas from five patients with Stevens-Johnson syndrome were studied immunohistochemically for several cell surface antigens and two cytokines. Chemical injury specimens were also studied.

Results-In all cases, immunohistochemistry revealed $\mathrm{LFA}-1, \mathrm{CD} 4, \mathrm{CD} 8$, and CD68 on subepithelial infiltrating cells. Also, HLA-DR and ICAM-1 were found on the surfaces of epithelial cells, subepithelial infiltrating cells, subepithelial fibroblasts, and endothelial cells in blood vessels. IFN- $\gamma$ was found in basal epithelial cells; subepithelial cells and subepithelial extracellular matrix CD19 and IL4 were not detected.

Conclusions-The infiltrating cell population in the Stevens-Johnson syndrome samples includes macrophages, CD4 positive $T$ cells, and CD8 positive $T$ cells. The cytokine expression pattern suggests CD4 positive $T$ cells are Th1 cells. The infiltrating cell population is similar in StevensJohnson syndrome and chemical injury conjunctivalised corneas.

(Br f Ophthalmol 2000;84:1191-1193)
\end{abstract}

Stevens-Johnson syndrome, first reported in $1922,{ }^{1}$ is an acute inflammatory disease that predominantly affects skin and mucosal membranes including the ocular surface. In the acute phase, ocular manifestations include corneal ulceration and severe pseudomembranous conjunctivitis. $^{2-5}$ After the initial attack has passed, about half of the patients with severe systemic Stevens-Johnson syndrome continue to have ocular surface problems that include symblepharon, entropion, ectropion, trichiasis, dry eye, persistent conjunctival inflammation, conjunctival injection, and corneal opacification. ${ }^{4}$

It is thought that the pathogenesis of Stevens-Johnson syndrome in the acute phase might be related to an immunological hypersensitivity to drugs or micro-organisms. ${ }^{6-8}$ However, little is known about the persistent inflammation during chronic phase StevensJohnson syndrome. To better understand the immunopathogenesis of the corneal conjunctivalisation that is seen in Stevens-Johnson syndrome, we examined tissue from five patients with Stevens-Johnson syndrome who presented at Kyoto Prefectural University of Medicine Hospital between March and July 1995. We similarly examined conjunctivalised corneas from three chemically injured eyes.

\section{Materials and methods}

Resected tissues specimens were frozen sectioned ( $6 \mu \mathrm{m}$ thick) and placed on glass slides. Immunohistochemical studies were performed by the standard ABC method. Cold acetone was used as fixative for the detection of LFA-1, CD4, CD8, CD19, CD68, HLA-DR, and ICAM-1 and 4\% paraformaldehyde was used as fixative for the detection of IFN- $\gamma$ and IL-4. To eliminate non-specific immune staining, $5 \%$ normal goat serum was used. All specimens were incubated overnight with the following primary antibodies at $4^{\circ} \mathrm{C}$ at the stated dilution (all were raised in mouse and purchased from Dako Corp (Glostrup, Denmark)): LFA-1 (1:200), CD4 (1:20), CD8 (1:200), CD19 (1:100), CD68 (1:100), HLA-DR (1:100), ICAM-1 (1:50). Antihuman IFN- $\gamma(50 \mathrm{mg} / \mathrm{ml})$ was purchased from Endogen (Cambridge, MA, USA) and the anti-human IL-4 $(5 \mathrm{mg} / \mathrm{ml})$ from Genzyme (Cambridge, MA, USA). For negative controls, normal mouse IgG was used instead of the primary antibody. Biotinylated rabbit antimouse immunoglobulin (Dako) was used as the secondary antibody, and specimens were incubated in it for 1 hour at a dilution of $1 / 800$, followed by the incubation in the avidin-biotinperoxidase complex (Vector Laboratories Inc, Burlingame, CA, USA) at room temperature for 30 minutes. In developing, DAB buffered with $0.05 \mathrm{M}$ TRIS buffer was used. Finally, sections were counterstained with methyl green.

\section{Results and discussion}

Before surgery, no inflammation was noted in patients 2 and 5 , whereas patients 1,3 , and 4 occasionally presented with mild inflammation. However, these mild inflammatory episodes invariably resolved without the administration of extra medication.

On histological examination, conjunctivalised corneas from all patients with StevensJohnson syndrome were characterised by fairly 
extensive subepithelial mononuclear infiltration (Fig 1A). No polymorphonuclear cells (PMNs) were evident in any regions of the specimens except for the vascular lumen. The conjunctivalised corneal epithelium was clearly thicker than normal in four of the five cases.

All specimens showed positive immunohistochemical reactions for anti-LFA-1 (Fig 1B), anti-CD4 (Fig 1C), anti-CD8 (Fig 1D), anti-CD68 (Fig 1F), anti-HLA-DR (Fig 1G), and anti-ICAM-1 (Fig 1H). HLA-DR and ICAM-1 immunoreactivities were detected on subepithelial infiltrating cells, epithelial cells and endothelial cells of blood vessels. LFA-1, CD4, CD8, and CD68 immunoreactivities were detected on subepithelial mononuclear infiltrating cells only. However, CD19 immu- noreactivity was not detected in any of the patients (Fig $1 \mathrm{E})$. IFN- $\gamma$ immunoreactivity was detected in subepithelial infiltrating cells, in the basal layer of the conjunctivalised corneal epithelium, and in endothelial cells of blood vessels (Fig 1I). IL-4 immunoreactivity was not evident except for on PMNs in the blood vessel (Fig 1J). Immunohistochemistry of conjunctivalised corneas from three chemically injured eyes (not shown) disclosed a similar pattern of cell surface antigen and cytokine immunoreactivity as did the conjunctivalised StevensJohnson syndrome corneas.

Stevens-Johnson syndrome is a self limited disease and many investigations have been done to elucidate the immunological mechanism of initial attack. ${ }^{6-8}$ Clinically, however,
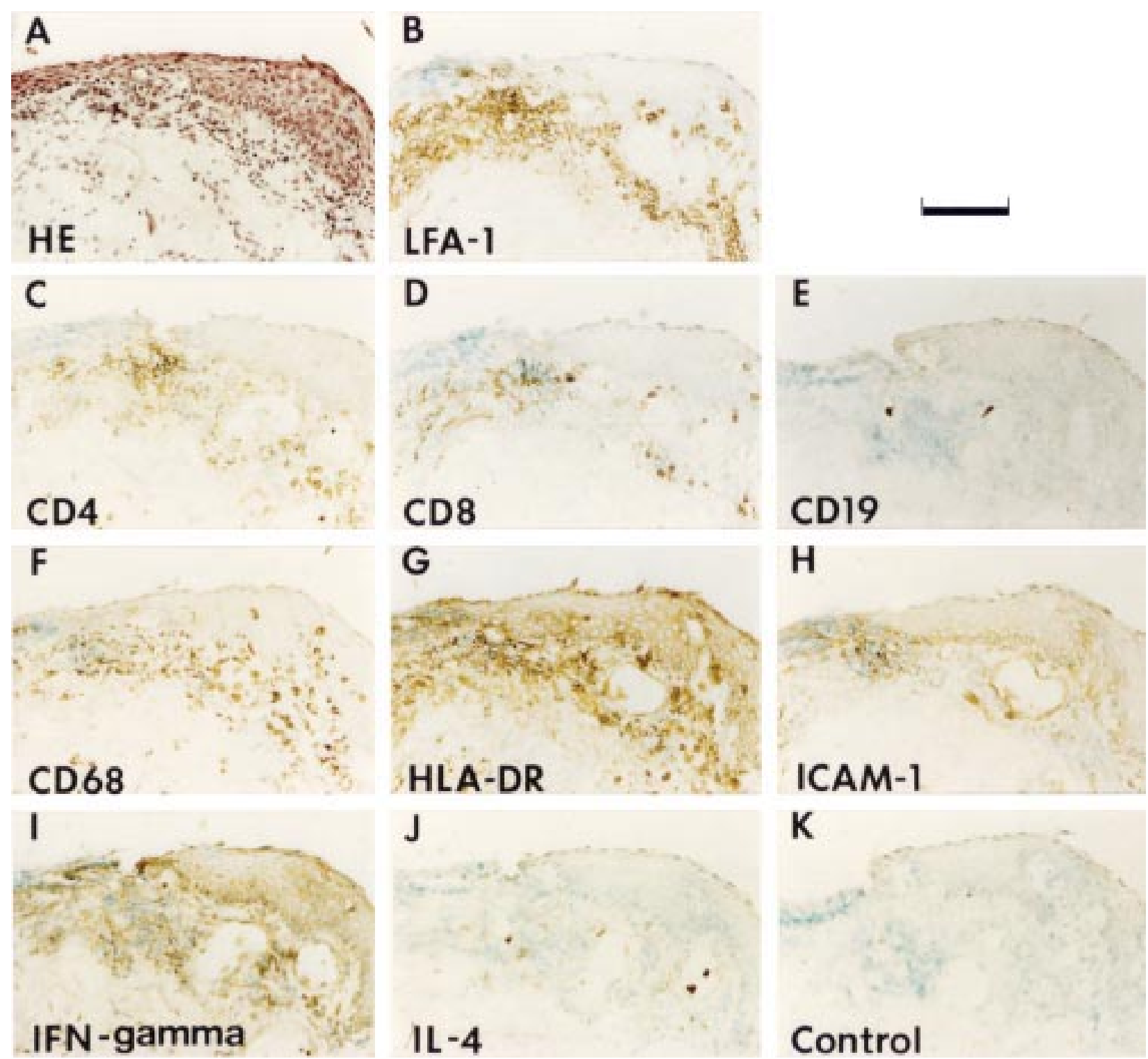

Figure 1 Representative serial sections from Stevens-fohnson conjunctiva (patient No 4). Immunohistochemical sections are stained by the ABC method with DAB (counterstained with methyl green). (A) Haematoxylin and eosin (HE). Conjunctivalised corneal epithelium moderately thickened and subepithelial infiltrating cells notable. (B) Anti-LFA-1. Many immunopositive cells seen in the substantia propria of the conjunctiva. (C) Anti-CD4. Immunopositive cells seen in the substantia propria of the conjunctiva. (D) Anti-CD8. Immunopositive cells seen in the substantia propria of the conjunctiva. (E) Anti-CD19. No positive staining is seen in this section except for some polymorphonuclear cells in the vascular lumen. (F) Anti-CD68. Many subepithelial infiltrating cells are immunostained. $(G)$ Anti-HLA-DR. Basal cells of conjunctival epithelium, subepithelial infiltrating cells, and endothelial cells of blood vessels are immunostained. (H) Anti-ICAM-1. Basal cells of conjunctival epithelium, subepithelial infiltrating cells, and endothelial cells of blood vessels are weakly immunostained. (I) Anti-IFN- $\gamma$. Basal cells of conjunctival epithelium, subepithelial infiltrating cells, and endothelial cells of blood vessels are weakly immunoreactive. (f) Anti-IL-4. No positive staining is seen in this section except for some polymorphonuclear cells in the vascular lumen. (K) A negative control section incubated with IgG1 in lieu of a primary antibody. Scale bar (upper right) $100 \mu \mathrm{m}$. 
Table 1 Patient clinical data and result of immunostaining

\begin{tabular}{|c|c|c|c|c|c|c|}
\hline & Patient 1 & Patient 2 & Patient 3 & Patient 4 & Patient 5 & $\begin{array}{l}\text { Immunoreactive } \\
\text { cells }\end{array}$ \\
\hline Age & 24 & 61 & 74 & 27 & 25 & \\
\hline Sex & Male & Male & Female & Male & Male & \\
\hline VA & $20 / 40$ & $20 / 400$ & $2 / 200$ & $\mathrm{HM}$ & $\mathrm{HM}$ & \\
\hline Duration & 11 years & 25 years & 30 years & 20 years & 3 years & \\
\hline Inflammation & Mild & None & Mild & Mild & None & \\
\hline LFA-1 & + & + & + & + & + & SI \\
\hline $\mathrm{CD} 4$ & + & + & + & + & + & SI \\
\hline CD8 & + & + & + & + & + & SI \\
\hline CD19 & - & - & - & - & - & \\
\hline CD68 & + & + & + & + & + & SI \\
\hline HLA-DR & + & + & + & + & + & SI, CCE, EV \\
\hline ICAM-1 & + & + & + & + & + & SI, CCE, EV \\
\hline IFN- $\gamma$ & + & + & + & + & + & SI, CCE, EV \\
\hline IL-4 & - & - & - & - & - & \\
\hline
\end{tabular}

Age $=$ age at surgery.

$\mathrm{VA}=$ visual acuity at the time of surgery.

Duration $=$ duration from the initial attack to surgery.

$\mathrm{HM}=$ hand movement.

Inflammation = clinical inflammation at the time of surgery

$+=$ immunoreactive.

$-=$ not immunoreactive, apart from some immunoreactive polymorphonuclear cells in vascular lumen.

SI = subepithelial infiltrating cells.

$\mathrm{CCE}=$ conjunctivalised corneal epithelial cells

$\mathrm{EV}=$ endothelial cells of blood vessel.

we often see patients with Stevens-Johnson syndrome with slowly progressive conjunctivalisation of cornea and decline of visual acuity even when many years have passed after the initial attack. Also, the outcome of keratoplasty for patients with Stevens-Johnson syndrome is very poor compared with other corneal diseases. Before this study, we believed that both poor surgical outcome and chronically progressive conjunctivalisation are based on the specific immunopathogenesis of StevensJohnson syndrome. To our surprise, substantial inflammatory cell infiltration was observed in the five patients with Stevens-Johnson syndrome, despite little or no inflammation being noted clinically. In contrast with our preconceptions, the immunopathological features of conjunctivalised corneas in Stevens-Johnson syndrome are not appreciably different from the immunopathological features of those in chemical injury. Also, within the group of five patients examined, the pattern of immunoreactivity to antibodies examined here was fairly consistent, and no clear correlation emerged between the immunopathological findings of an individual patient and the patients' clinical histories (Table 1). Our results revealed that inflammatory cells in the substantia propria of conjunctivalised corneas of patients with Stevens-Johnson syndrome are composed of CD4 positive $\mathrm{T}$ cells, CD8 positive $\mathrm{T}$ cells, and/or macrophages. B lymphocytes are probably not present. In addition to this, we tried to identify CD 4 positive $T$ cells from the cytokine expression pattern. Our results revealed the predominance of IFN- $\gamma$ suggesting that CD 4 positive $\mathrm{T}$ cells were mainly Th1 cells that can promote cellular immunity. ${ }^{9}$
Interestingly, Foster and associates, in their immunohistochemical study of two patients with Stevens-Johnson syndrome who experienced recurrent episodes of inflammation, found that the predominant $\mathrm{T}$ cells in conjunctiva were $\mathrm{T}$ helper cells, rather than suppressor $\mathrm{T}$ cells which usually outnumber $\mathrm{T}$ helper cells in normal conjunctiva. ${ }^{10}$ These authors also found HLA-DR positive cells in the epithelium and substantia propria of conjunctiva that they identified as Langerhans cells, macrophages, and activated $\mathrm{T}$ cells. Though their results are similar to ours, the clinical types of patients they examined are apparently different from ours. However, the difference between patients with Stevens-Johnson syndrome who experience recurrent episodes of conjunctival inflammation and those who don't is not readily evident.

A similar immunostaining pattern between Stevens-Johnson syndrome and chemical injury corneas suggests that inflammatory cell infiltration in the substantia propria of patients with Stevens-Johnson syndrome may not be a primary reaction but a secondary reaction from other ocular abnormalities related to stem cell failure such as trichiasis or severe dry eye. We feel that these pre-existing infiltrating cells at surgery may play an important part in graft rejection and/or post-surgically induced inflammation in patients with Stevens-Johnson syndrome.

The authors thank Nigel Fullwood, $\mathrm{PhD}$, for critical reviewing of the manuscript.

Supported by grants in aid for scientific research (numbers 09771460, 09877336, and 08457467) from the Ministry of Education, Culture and Science of Japan, Tokyo, Japan, by the Kyoto Foundation for the Promotion of Medical Science, Kyoto Foundation for the Promotion of Medical Science,
Kyoto, Japan, and by the Intramural Research Fund of Kyoto Kyoto, Japan, and by the Intramural Research F
Prefectural University of Medicine, Kyoto, Japan.

1 Stevens AM, Johnson FC. A new eruptive fever associated with stomatitis and ophthalmia. Am $\mathcal{f}$ Dis Child with stomatitis

2 Howard GM. The Stevens-Johnson syndrome. Am $\mathcal{F}$ Ophthalmol 1963;55:893-900.

3 Patz A. Ocular involvement in erythema multiforme. Arch Ophthalmol 1950;43:244-56.

4 Dohlman $\mathrm{CH}$, Doughman DJ. The Stevens-Johnson syndrome. Trans New Orleans Acad Ophthalmol 1972;24: 236-52.

5 Arstikaitis MJ. Ocular aftermath of Stevens-Johnson syndrome. Arch Ophthalmol 1973;90:376-9.

6 Bushkell LL, Mackel SE, Jordon RE. Erythema multiforme: direct immunofluorescence studies and detection of circulating immune complexes. F Invest Dermatol 1980;74:3724.

7 Kazmierowski JA, Wuepper KD. Erythema multiforme: mmune complex vasculitis of the superficial cutaneous microvasculature. F Invest Dermatol 1978;71:366-9.

8 Wuepper KD, Watson PA, Kazmierowski JA. Immune complexes in erythema multiforme and the Stevens-Johnson syndrome. F Invest Dermatol 1980;74:368-71.

9 Mosmann TR, Cherwinski H, Bond MW, et al. Two types of murine helper T cell clone. I. Definition according to profiles of lymphokine activities and secreted proteins. 7 Immunol 1986;136:2348-57.

10 Foster CS, Fong LP, Azar D, et al. Episodic conjunctival inflammation after Stevens-Johnson syndrome. Ophthalmology 1988;95:453-62. 